\title{
Pathogen-Derived Oligosaccharides Improve Innate Immune Response to Intracellular Parasite Infection
}

\author{
Alex Osanya, ${ }^{*}$ Eun-Ho Song, ${ }^{\dagger}$ Kyle Metz, ${ }^{*}$ \\ Raeann M. Shimak, ${ }^{*}$ Paola Mercedes Boggiatto, ${ }^{*}$ \\ Elise Huffman, ${ }^{*}$ Charles Johnson, ${ }^{*}$ \\ Jesse M. Hostetter, ${ }^{*}$ Nicola L.B. Pohl, ${ }^{\dagger}$ and \\ Christine A. Petersen* \\ From the Department of Veterinary Pathology,* College of \\ Veterinary Medicine, and the Department of Chemistry, ${ }^{\dagger}$ College \\ of Liberal Arts and Sciences, Iowa State University, Ames, Iowa
}

Pathogen glycolipids, including Leishmania spp. lipophosphoglycan (LPG) and Mycobacterium tuberculosis mannosylated lipoarabinomannan (ManLAM), modulate essential interactions with host phagocytic cells. Polysaccharide and lipid components promote immunomodulation. Owing to the stereochemistry required to synthesize oligosaccharides, the roles for oligosaccharides in the pathogenesis of infectious diseases have remained largely unknown. Recent advances in carbohydrate chemistry allowed us to synthesize pathogen surface oligosaccharides to discern their immune response-altering activities. Trimannose cap carbohydrates from ManLAM and LPG altered the production of proinflammatory cytokines via a toll-like receptor (TLR2)-mediated mechanism in vitro and in vivo. In vivo treatment with trimannose led to increased Th1-polarizing, IL-12p40-producing cells from the draining lymph nodes of treated Leishmania majorinfected mice compared with cells from untreated infected mice. Trimannose treatment increased the production of other Th1 proinflammatory cytokines (ie, interferon- $\gamma$, IL-6, and tumor necrosis factor- $\alpha$ ) critical for a productive immune response to either pathogen. This significant difference in cytokine production between trimannose cap sugar-treated and control groups was not observed in draining lymph node cells from TLR2 $^{-/-}$mice. Type of inflammation and rate of bead entry into macrophages and dendritic cells were different for trimannose-coated beads compared with control oligosaccharide-coated beads, indicating selective lectin receptor/oligosaccharide interactions mediating cell entry and cytokine production. These novel findings may prompt the development of targeted oligosaccha- ride adjuvants against chronic infections. (Am J Pathol 2011, 179:1329-1337; DOI: 10.1016/j.ajpath.2011.05.053)

Pathogens that cause chronic infectious diseases often inhabit host phagocytic cells. Detection of pathogens and initiation of the innate immune response involve interactions between conserved motifs in the ligands as pathogen-associated molecular patterns and in pattern recognition receptors, including toll-like receptors (TLRs) and C-type lectin receptors expressed on host phagocytic cells. ${ }^{1-3}$ Macrophages serve as a predominant phagocytic cell type specialized for identification, phagocytosis, and destruction of invading pathogens. Binding of microbial ligands to pattern recognition receptor triggers various crucial immune effector functions, including the production of proinflammatory cytokines. Glycolipids are major ligands whose components have been shown to induce immune modulation. ${ }^{4-6}$

Carbohydrate chains are abundantly expressed on outer surfaces of most bacterial, protozoan, viral, and fungal pathogens. These chains are without peer in structural diversity. Leishmania parasites are covered by a complex glycocalyx whose glycoconjugate components are thought to be important factors in promoting viru-

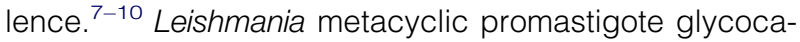
lyx contains several phosphoglycans composed of polymeric disaccharide phosphate repeating units. ${ }^{11}$ One of the most abundant promastigote surface glycolipids on Leishmania spp. is lipophosphoglycan (LPG), containing 15 to 30 repeating units, which distally bears an external cap oligosaccharide and proximally is anchored to the parasite membrane by glycosyl phosphatidyl inositol. ${ }^{12}$ Many studies have shown that purified LPG has a significant effect on

Supported by the National Institutes of Health (R21AI074711 to C.A.P).

Accepted for publication May 31, 2011.

Current address of A.O., Boehringer Ingelheim Vetmedica Inc., Ft. Dodge, lowa; of E.-H.S., Department of Bioengineering, University of Washington, Seattle, Washington; of K.M., Bloomberg School of Public Health, Johns Hopkins University, Baltimore, Maryland; and of P.M.B., Department of Microbiology, University of lowa, lowa City, lowa.

Address reprint requests to Christine A. Petersen, D.V.M., Ph.D., 2714 Vet Med, Department of Veterinary Pathology, College of Veterinary Medicine, lowa State University, Ames, IA 50011. E-mail: kalicat@iastate.edu. 
parasite survival, attributed to suppression of host signaling and evasion of activated complement. ${ }^{7-9}$ Multiple elegant genetic studies altering Leishmania glycolipid expression identified that these molecules are important for parasite virulence and persistence, although there are critical species differences regarding the complex multiple enzymes required to assemble a virulent parasite glycocalyx. ${ }^{13}$ Pathogenic species of Mycobacterium evolved similar strategies to establish long-term infection. The unique composition of the mycobacterial multiglycosylated envelope complex lipoglycans mannosylated lipoarabinomannan (ManLAM) and lipomannan, strategically located at the host-pathogen interface, contributes to immune alteration. ${ }^{14-16}$ The three distal glycosylated portions of ManLAM were shown to promote mycobacterial binding to the lectin binding receptor DC-SIGN and to promote mycobacterial virulence. ${ }^{14}$ LPG and ManLAM, implicated in modulating inflammatory responses and supporting continuation of chronic disease, ${ }^{17}$ provide the cap sugars of focus for this study.

In this study, we use unit oligosaccharide carbohydrates coating inert latex beads as a novel model system to determine the immunologic activity of subunit sugars. We used five cap sugars found on Mycobacterium tuberculosis and Mycobacterium bovis ManLAM, Leishmania mexicana and Leishmania major promastigote LPG, in vitro noninfective mutant Leishmania donovani LPG, and inert control sugar lactose to identify the ability of different oligosaccharides to alter the innate immune response. We found that trimannose, found on mycobacterial ManLAM and Leishmania spp. LPG, alters the production of IL-12p40 in macrophages in vitro and in vivo by engaging the TLR2 pathway. When introduced into mice, the trimannose-coated bead had increased numbers of IL12p40-producing cells and production of proinflammatory cytokines in wild-type but not TLR2 ${ }^{-1-}$ mice. Understanding how subunit oligosaccharides, singularly or combined, produce immune alteration aids in the production of responsive targeted immunostimulants against complex pathogens, including M. tuberculosis and Leishmania.

\section{Materials and Methods}

\section{Cells and Cell Culture}

Murine macrophage cell line J774.A1 (American Type Culture Collection, Rockville, MD) was cultured in macrophage media (Dulbecco's modified Eagle's medium, $20 \mathrm{mmol} / \mathrm{L}$ HEPES, pH 7.3 to 7.4 , supplemented with $10 \%$ heat-inactivated fetal bovine serum, $100 \mathrm{U} / \mathrm{mL}$ of penicillin, and $100 \mu \mathrm{L} / \mathrm{mL}$ of streptomycin) at $37^{\circ} \mathrm{C}$ in $5 \% \mathrm{CO}_{2}$. Bone marrow-derived macrophages were obtained as previously as described. ${ }^{18}$ Briefly, femur and tibia bone marrow cells were extracted and plated with $30 \mathrm{~mL}$ of macrophage media at $37^{\circ} \mathrm{C}$ in $5 \% \mathrm{CO}_{2}$. On day 7 , nonadherent cells were removed and adherent cells were harvested. Live cells were counted via Trypan blue exclusion and were resuspended in complete tissue culture medium for further experimentation.

\section{Generation of Cap Sugar-Coated Beads}

Cap sugar-coated beads (CSCBs) were synthesized as described previously. ${ }^{19}$ Briefly, 1- $\mu \mathrm{m}$ fluorescein isothiocyanate-labeled latex beads (Sigma-Aldrich, St Louis, $\mathrm{MO}$ ) were linked to each oligosaccharide via amide linkage. Pathogen-derived oligosaccharides were selected for bead attachment and further investigation based on previous studies describing distal cap carbohydrate moieties from Leishmania and Mycobacterium spp. ${ }^{11,17}$ To N link oligosaccharides to commercially produced beads, approximately $50 \mathrm{Eq}$ of each synthesized cap sugar was used for each coupling reaction. A total of $3.5 \times 10^{-4}$ $\mathrm{mmol}$ of cap sugar was required to attain $100 \%$ coverage of a single $1-\mu \mathrm{m}$ latex bead. Carbohydrate linkage was tested via Kaiser colorimetric assay, ${ }^{20}$ and a phenolsulfuric acid assay was used to confirm the sugar density on each bead. ${ }^{21}$ Beads were normalized to $2.65 \times 10^{10}$ beads $/ \mathrm{mL}$ in sterile water at $4^{\circ} \mathrm{C}$.

\section{Macrophage Treatments and Latex CSCBs}

Bone marrow-derived macrophages and J774.A1 cells were plated in 24-well plates with glass coverslips at a density of $5 \times 10^{5}$ cells $/ \mathrm{mL}$. After 24 hours, macrophages were activated with $100 \mathrm{U} / \mathrm{mL}$ of interferon- $\gamma$ (IFN- $\gamma$; BD Pharmingen, San Diego, CA) and $100 \mathrm{ng} / \mathrm{mL}$ of lipopolysaccharide (LPS) (Escherichia coli J5; Sigma-Aldrich), followed by the addition of CSCBs at multiplicity of infection of 5:1 beads per cell. Uptake and internalization were assessed via microscopy, and bead entry into cells was not statistically different between bead types (data not shown). Blockade of TLR2 and mannose receptor (MR) on the surface of J774.A1 cells was mediated through the addition of extracellular domain blocking antibodies [anti-human/ mouse CD282 (TLR2), clone T2.5 (eBioscience, San Diego, CA), $10 \mu \mathrm{g} / \mathrm{mL}$, and anti-mouse CD206 (MR), clone MRSD3 (BioLegend, San Diego, CA), $20 \mu \mathrm{g} / \mathrm{mL}$ ] for 2 hours, followed by the addition of CSCBs for 16 hours. Control nonspecific antibody responses were analyzed via the addition of mouse lgG1 isotype for TLR2 $(20 \mu \mathrm{g} / \mathrm{mL})$ and rat antimouse IgG2a (eBioscience) for CD206/MR (20 $\mu \mathrm{g} / \mathrm{mL})$.

\section{IL-12p40 ELISA}

Supernatants from bone marrow-derived macrophages and J774.A1 cultures were harvested at various time points after bead treatment. IL-12p40 enzyme-linked immunosorbent assay (ELISA) was performed using commercially available purified IL-12p40 and biotinylated anti-IL-12p40 antibodies (BD Pharmingen), peroxidase-conjugated streptavidin (Jackson ImmunoResearch Laboratories Inc., West Grove, PA), and ABTS microwell peroxidase substrate (Roche, Indianapolis, IN).

\section{Luminex Assay}

For in vitro Luminex analysis, cells were cultured in triplicate, and draining lymph node (DLN) cells from in vivo experiments were cultured in duplicate; cell-free supernatants were harvested after 72 hours of culture and then 
were analyzed for the concentration of tumor necrosis factor- $\alpha$ (TNF- $\alpha$ ), IFN- $\gamma$, IL-6, IL-4, IL-10, and IL-12p40 (in vitro analysis) and TNF- $\alpha$, IFN- $\gamma$, IL-6, IL-4, IL-10, IL-17, leukemia inhibitory factor, IL-5, and IL-13 (ex vivo analysis) using multiplexed flow cytometric assay (Luminex, Austin, TX). For in vitro analysis, $50 \mu \mathrm{L}$ of bead suspension was added to each well and was washed. Fifty microliters of sample and standard were added to each well, plate sealed, and shaken for 30 seconds at 1100 $\mathrm{rpm}$ and then were incubated for 1 hour at $300 \mathrm{rpm}$ at room temperature and washed; $25 \mu \mathrm{L}$ of prediluted detection antibody was added and mixed. The plate was incubated for 30 minutes at $300 \mathrm{rpm}$ in the dark. After washing, $50 \mu \mathrm{L}$ of $1 \times$ streptavidin-phosphatidylethanolamine was added to each well and incubated for 10 minutes. Plate was washed and resuspended in $125 \mu \mathrm{L}$ of the assay buffer, sealed, mixed, and immediately read. Ex vivo samples were processed using a Milliplex MAP mouse cytokine/chemokine kit (Millipore, Billerica, MA) as described in the package insert. Samples were incubated with the beads for 2 hours at room temperature followed by $\mathrm{O} / \mathrm{N}$ incubation at $4^{\circ} \mathrm{C}$, and samples were acquired using the Luminex default settings. Median fluorescent intensity data were analyzed using the fiveparameter logistic curve fitting method for calculating cytokine and chemokine concentrations.

\section{IL-12 EL/spot Analysis}

IL-12 ELIspots were performed on DLN cells. Immulon 2 plates (Fisher, Fair Lawn, NJ) were coated with $5 \mu \mathrm{g} / \mathrm{mL}$ of IL-12 (R\&D Systems, Minneapolis, MN) overnight at $4^{\circ} \mathrm{C}$. After washing with PBS, commercially available biotinylated anti-IL-12p40 antibodies (BD Pharmingen) were added at a 1:10,000 dilution in 5\% fetal bovine serum overnight at $4^{\circ} \mathrm{C}$. ELIspots were developed using 2-amino-2-methyl-1-propanol (ICN Biomedicals Inc., Aurora, $\mathrm{OH}$ ) and 5-bromo-4-chloro-3-indoly-phosphate (Fisher) and were counted within 24 hours.

\section{In Vivo Bead Treatment and L. major Footpad Infection}

Five mice per experimental group were injected with CSCBs and infected with L. major in the footpad. Mice were humanely euthanized at time 0 (2 hours), 24 hours, and 48 hours; footpads were processed for histopathologic analysis, and DLNs were processed for IL-12p40 ELIspot and Luminex analysis. All experiments involving animals were performed as approved by the lowa State University Institutional Animal Care and Use Committee.

\section{Tissue Processing and Analysis}

From each experimental group ( $n=5)$, after humane euthanasia, infected footpads from three mice were processed for immunohistochemical analysis to classify bead-containing cells as $\mathrm{C} 11 \mathrm{~b}^{+}, \mathrm{CD} 11 \mathrm{c}^{+}$, non-CD11 $\mathrm{b}^{+}$, or non-CD11 ${ }^{+}$for histologic characterization of the inflammatory response. Entire cross sections of each foot- pad from three mice per treatment group were evaluated for degree of cellular inflammation. Frozen sections of each footpad were sectioned at $5 \mu \mathrm{m}$, stained with H\&E for histopathologic evaluation and immunofluorescent la-

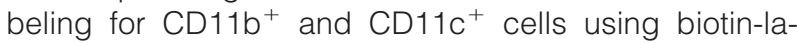
beled anti-mouse CD11b and CD11c antibodies (eBioscience), followed by Cy3-conjugated streptavidin (Jackson ImmunoResearch Laboratories Inc.). All the slides were examined, counted, and described by a board-certified veterinary pathologist. At least 20 fields $(40 \times)$ were evaluated per treatment group. The degree of cellular inflammation was assessed via a rising scale from 0 to 3 , with a score of 0 representing no inflammation; 1 , small numbers of inflammatory cells; 2 , moderate numbers of inflammatory cells; and 3, large numbers of inflammatory cells. Percentage of bead-positive CD11b/c was determined by counting the number of fluorescent beads that co-localized with a CD11b- or CD11c-positive cell relative to the total number of beads counted.

\section{Statistical Analysis}

Statistical analysis was assessed by one-way analysis of variance and between two values was determined via a post-analysis of variance Tukey's multiple comparison test. $P<0.05$ was considered statistically significant. This difference was confirmed via Student's t-test.

\section{Results}

\section{Cap Sugars Are Biologically Available on Coated Latex Beads}

Previous studies have demonstrated that the highly glycosylated distal third of LPG and ManLAM confer and exhibit immunomodulatory activity. ${ }^{10,12,14-16}$ More recently, distal cap sugars were shown to lead to differential virulence ${ }^{17}$ and alterations in T-cell responses, ${ }^{22}$ but activity could not be attributed to oligosaccharide alone either because purification of the cap sugars from a heterogeneous population of small saccharides present at the end of larger glycoconjugates leads to a muddle of many different sugars or could be demonstrated only after conjugation of oligosaccharide(s) to peptide antigens. We overcame these problems by developing novel automated synthesis processes to build pathogen-derived oligosaccharides and amide link them to fluorescein isothiocyanate-conjugated latex beads. ${ }^{19}$ Four different pathogen CSCBs, with uncoated beads and inert lactose-coated beads as negative controls, provided a starting point from which to define specific biologic responses to these pathogen-derived oligosaccharides (Figure 1). These pathogen-derived and virulence-related oligosaccharides included $\alpha$-linked trimannose (Figure 1D), previously shown to cap ManLAM of $M$. tuberculosis ${ }^{17}$ and LPG of L. donovani. ${ }^{11}$ Loss of this oligosaccharide cap from $M$. bovis bacillus Calmette-Guerin and M. tuberculosis ManLAM was shown to reverse inhibition of LPS-induced IL-12 production by human dendritic cells. ${ }^{23}$ We demonstrated the chemical composition and surface availability of these latex bead-bound cap sugars. ${ }^{19}$ Given 


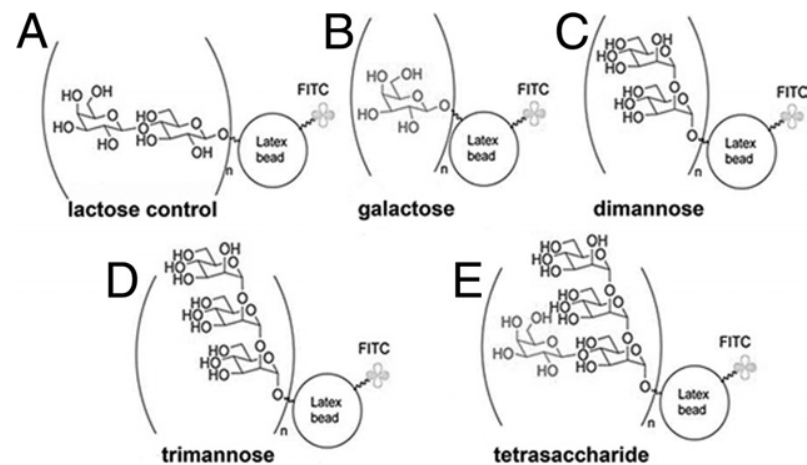

Figure 1. Diagram of pathogen-derived carbohydrate-coated beads. The chemically synthesized sugars lactose, galactose, dimannose, trimannose, and tetrasaccharide were covalently linked to fluorescein isothiocyanate (FITC)-conjugated latex beads. These cap sugars were selected based on their presence on the surface of Leishmania and/or Mycobacterium. A Lactose inert sugar control. B: Galactose isolated cap sugar from noninfectious L. donovani LPG. C: Dimannose cap sugar on L. major and L. donovani LPG. D: Trimannose isolated cap sugar on $M$. tuberculosis ManLAM and $L$. donovani. E: Tetrasacharide cap sugar on $L$. donovani and L. mexicana LPG.

previous results regarding the effect of the larger pathogen glycoconjugates ManLAM and LPG on critical cytokine production by macrophages and dendritic cells, we used novel bead-linked oligosaccharides to determine the role of these five pathogen-derived cap sugars alone in altering in vitro macrophage cytokine production

\section{Oligosaccharide Pathogen Cap Sugars Promote Differential Cytokine Production in Vitro}

IL-12 is the primary cytokine for initiation of a Th1 immune response. ${ }^{24-26}$ A Th1 response is necessary for in vitro and in vivo removal of intracellular Leishmania and Mycobacterium spp. ${ }^{23,27-30}$ Based on the importance of IL-12 in the in vitro removal of Leishmania and Mycobacterium spp., we tested the ability of CSCBs to drive differential cytokine responses through measurement via ELISA of IL-12p40 production in bead-treated $\mathbf{J 7 7 4}$ cells. LPS/IFN- $\gamma$ stimulated $\mathrm{J} 774$ cells exposed to each CSCB induced three alternative responses (Figure 2, A and B). Treatment of $\mathrm{J} 774$ cells with trimannose-coated beads led to significantly decreased IL-12p40 production after activa- tion with IFN- $\gamma$ and LPS (Figure 2A) compared with control IFN- $\gamma$ and LPS-stimulated cells. Dimannose- and galactose-coated beads increased IL-12p40 production above that observed in cells stimulated with IFN- $\gamma$ and LPS (Figure 2B). Uncoated beads and beads coated with lactose, a relatively inert sugar, did not significantly change IL-12p40 production from that of control IFN- $\gamma$ and LPS-stimulated $\mathrm{J} 774$ cells (Figure 2, A and B). A similar profile of IL-12p40 production was found using bone marrow-derived macrophages. Rate of bead entry was determined via microscopy and was found to be similar for all bead treatments.

To identify whether the differential cytokine production pattern observed after bead treatment was specific to IL-12p40, we analyzed the production of multiple cytokines via Luminex assay. We observed altered proinflammatory cytokine production due to bead treatment in J774 cells, most significantly TNF- $\alpha$ and IL-6 (Figure 2, C and $\mathrm{D})$. There were no significant differences in the production of cytokines not expected to be produced by in vitro macrophages: IL-4, IL-10, and IFN- $\gamma$ (data not shown). Alteration of macrophage cytokine production by these simple bead-linked oligosaccharides demonstrates that alteration of macrophage cytokine responses by more complex glycolipids, including ManLAM and LPG, is likely to be partially due to alteration by subunit distal cap oligosaccharides. Based on the observed different responses from these cap oligosaccharides, we predicted that these differences may be mediated through varied C-type lectin receptors.

\section{Cap Sugars Derived from ManLAM Are Required TLR2 to Elicit IL-12p40 Suppression in Macrophages}

Production of IL-12p40 and proinflammatory cytokines by macrophages is initiated by engagement of lectin-binding receptors by one or multiple carbohydrate-bearing ligands found on complex pathogen surfaces. ${ }^{1,31,32}$ Previous studies have shown that mannose-containing glycolipids induce IL-12 production in a manner different from classical TLR ligands, ${ }^{33}$ whereas ManLAM was
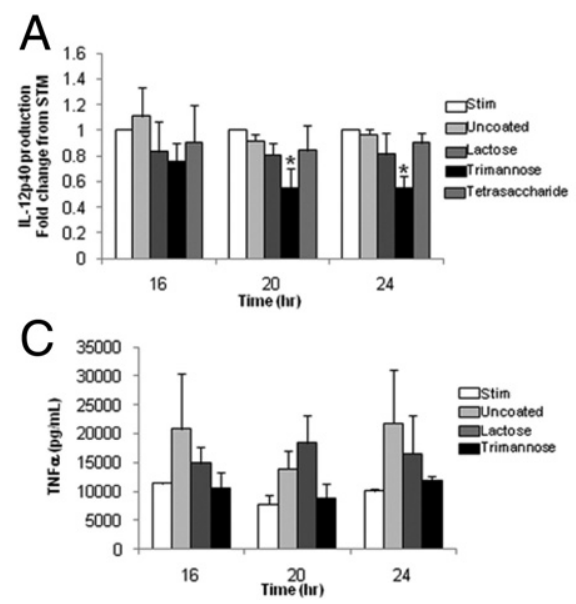

政
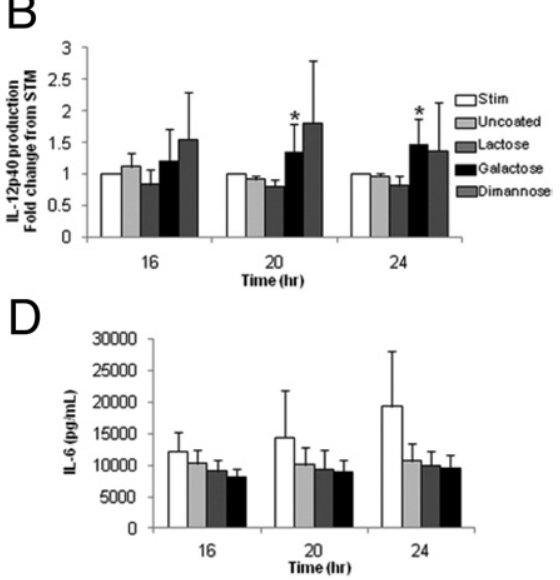

Figure 2. Latex CSCBs differentially modulated the production of TNF- $\alpha$, IL- 6 , and IL-12p 40 . $\mathrm{J} 774$ cells were stimulated with $50 \mathrm{ng} / \mathrm{mL}$ of LPS and $200 \mathrm{IU} / \mathrm{mL}$ of IFN- $\gamma$ and were exposed to various CSCBs as indicated. Supernatants were collected 16, 20, and 24 hours after bead exposure for ELISA (IL-12p40) (A and B) and Luminex analysis (C, TNF- $\alpha$; D, IL-6). A: IL-12p40 ELISA from $J 774$ cells stimulated with LPS and IFN- $\gamma$ and exposed to uncoated, inert lactose-coated, trimannose-coated, and tetrasaccharide-coated beads. B: IL-12p40 ELISA from J774 cells stimulated with LPS and IFN- $\gamma$ and exposed to un coated, inert lactose-coated, galactose-coated, and dimannose-coated beads increased production over stimulated cells alone. C: Luminex analysis for TNF- $\alpha$ production. D: Luminex analysis for IL-6 production. All the data were from three different experiments. Error bars depict SD. ${ }^{*} P \leq 0.05$ via Student's $t$-test. 


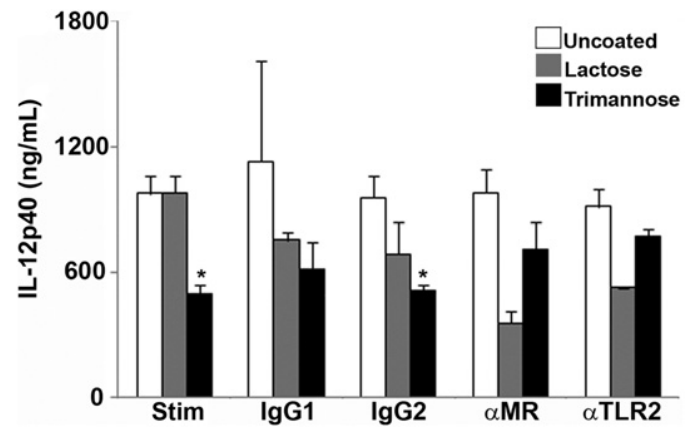

Figure 3. Inhibition of macrophage IL-12p40 secretion by trimannosecoated latex beads is TLR2 and MR dependent. J774 cells were activated with LPS and IFN- $\gamma$ for 2 hours, washed, and incubated in the presence or absence of various anti-pattern recognition receptor antibodies or their respective control isotype(s) as indicated for 2 hours followed by exposure to CSCBs as indicated. Supernatants were collected 24 hours after bead exposure and were used for IL-12p40. Data from three independent experiments in duplicate. Error bars depict SD. ${ }^{*} P \leq 0.05$ via Student's $t$-test.

shown to inhibit the production of IL-12 in human dendritic cells via an MR-dependent mechanism that interfered with TLR-based responses. ${ }^{23}$ We examined IL$12 \mathrm{p} 40$ production after the addition of TLR2 and MR blocking antibodies. The addition of TLR2 blocking antibodies during the treatment of activated $\mathrm{J} 774$ cells with trimannose-coated beads eliminated the reduction of IL12 p40 production (Figure 3). In comparison, this treatment did not alter the production of IL-12p40 from cells treated with lactose-, galactose-, or dimannose-coated beads and TLR2 blocking antibodies compared with control isotype antibody treatment (data not shown). These data indicate that a TLR2-mediated pathway is involved in trimannose-mediated reduction of activated macrophage IL-12p40 production. Based on previous findings described for the treatment of dendritic cells with whole ManLAM, we were interested in determining whether a similar response could be found after the addition of MR blocking antibodies to trimannose-capped bead-treated cells. ${ }^{23}$ We found that the addition of MR blocking antibodies recovered IL-12p40 production to a level similar to that observed after TLR2 blocking antibody production (Figure 3), indicating that this may be a response that requires a balance of signaling through both $\mathrm{C}$-type lectin receptors. Based on this diverse range of in vitro macrophage responses to pathogen-derived oligosaccharidecoated beads, we were interested in determining whether these responses would recapitulate when used during in vivo exposure to antigen-presenting cells.

\section{In Response to L. major Infection, Trimannose Bead-Treated Mice Increase Production of Proinflammatory Cytokines in a TLR2-Dependent Manner}

Previous studies have determined that dendritic cell presentation of T-cell epitopes modulated via glycosylation led to significantly enhanced presentation $22,34,35$ and more robust $\mathrm{T}$-cell effector function. ${ }^{22}$ Based on the present findings that different CSCBs could enhance or reduce cytokine production, we hypothesized that these bead-linked oligosaccharides would differentially alter cytokine production from DLN cells when given at the time of infection with an intracellular pathogen, L. major. Five mice per group were infected in the footpad with $L$. major alone or were provided with a 10:1 ratio of CSCBs to parasites. Mice were sacrificed at time 0 and 24 and 48 hours after infection and treatment, and cytokine production was measured via Luminex assay or ELIspot (IL-12p40) using supernatants from ex vivo DLN cells (Figure 4). To prime cytokine production specifically in response to oligosaccharide-coated beads, we included a comparison of conditions where we added additional beads or control $L$. major antigen to each respective well (cognate stimulation; Figure 4). In vivo treatment of mice with trimannose in conjunction with $L$. major infection led to robust increases in IL-12p40-producing DLN cells (Figure 4A) and increased DLN cell production of IL-6, TNF- $\alpha$, and IFN- $\gamma$ proinflammatory cytokine production (Figure 4B) compared with L. major infection alone, L. major infection and treatment with uncoated beads or beads coated with lactose. The addition of L. major freeze-thaw antigen to DLN cell culture (L. major cognate stimulation; Figure 4) robustly increased produc-

A

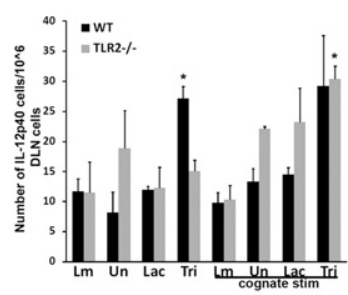

B
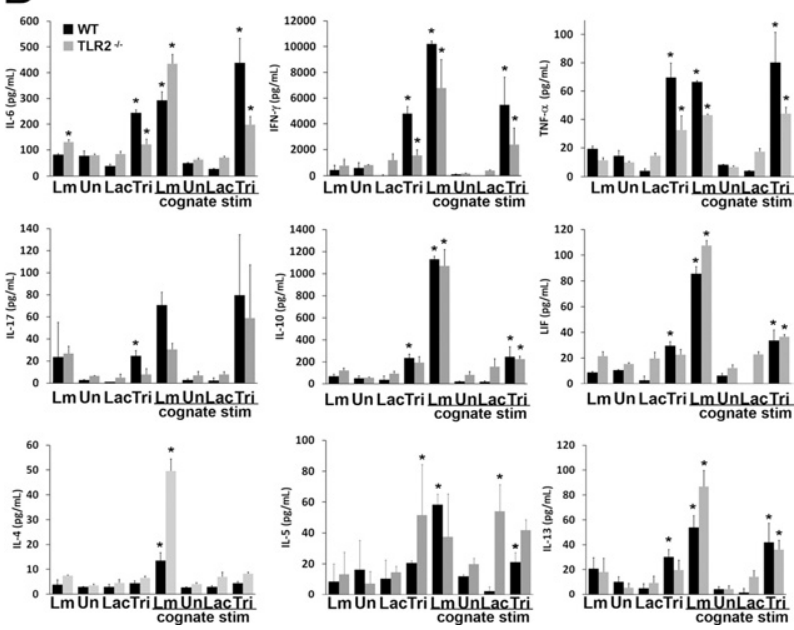

Figure 4. ELIspot and Luminex analysis reveals differential cytokine production by CSCBs compared with $L$. major infection alone. Mice were infected with $1 \times 10^{6}$ L. major promastigotes alone or in conjunction with $1 \times 10^{7} \mathrm{CSCBs}$. Five mice per experimental group were sacrificed at 48 hours and were processed for IL-12p40 ELIspot (A) and Luminex (B) cytokine analyses. Supernatants from DLN cells from mice infected and treated as labeled (five mice per group) were cultured for 72 hours ex vivo. To promote oligosaccharide-coated bead-specific responses compared with $L$. major responses, after plating, DLN cells were stimulated with either beads or $L$. major antigen as indicated (cognate stim). B: Of 32 cytokines analyzed, production is shown for proinflammatory cytokines IL-6, IFN- $\gamma$, and TNF- $\alpha$ (top row); other significant cytokines IL-17 and IL-10 and leukemia inhibitory factor (LIF) (middle row); and Th2 cytokines IL-4, IL-5, and IL-13 (bottom row). Error bars depict SD. ${ }^{*} P \leq 0.05$ via analysis of variance and Tukey's posttest. Lac, lactose coated; Lm, L. major infected; Tri, trimannose coated; Un, uncoated; WT, wild type. 


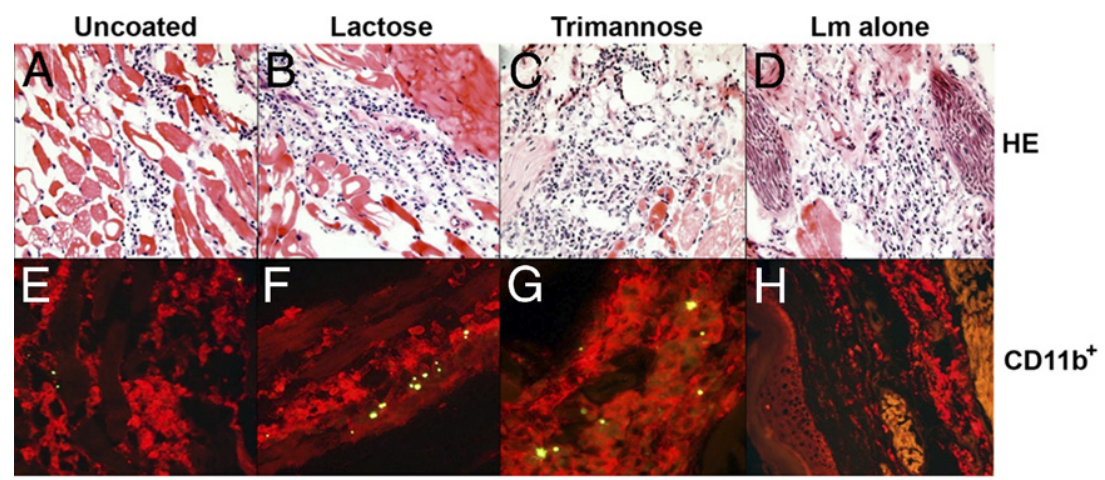

Figure 5. Treatment of mice with CSCBs in conjunction with L. majorfootpad infection leads to differential innate immune responses. Mice were infected with $1 \times$ $10^{6} \mathrm{~L}$. major promastigotes alone or in conjunction with $1 \times 10^{7} \mathrm{CSCBs}$. Five mice per experimental group were sacrificed at 0,24 , and 48 hours, and footpads were processed for immunofluorescence and histopathologic analysis. A-D: H\&E-stained tissue sections from uncoated (A), lactose-coated (B), trimannosecoated (C), and L. major-infected (D) footpads were scored for the degree of cellular inflammation (I) as described in Materials and Methods. E-H: Fluorescein isothiocyanate-labeled beads and CD $11 \mathrm{~b}^{+}$cells were visualized via fluorescence microscopy, and the percentage of beads found in each cell type was counted (J). Error bars represent SEM of cells counted in at least 20 high-power fields per treatment group. ${ }^{*} P<0.05$ via Student's $t$-test compared with uncoated beads (I $P=0.007)$ or uncoated and lactose-coated beads ( $\mathbf{J}$ $P=0.0016$ and $P=0.0027$, respectively). Lac, lactose coated; Lm, L. major infected; ND, not determined; Tri, trimannose coated; Un, uncoated; WT, wild type.

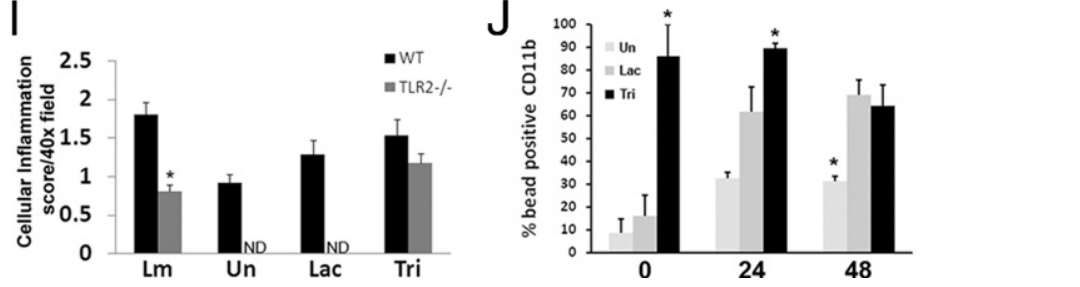

cells contained these beads and in identifying the character and amount of inflammation found at the site of infection and bead treatment. Trimannose-coated beadtreated L. major-infected footpads and L. major-infected alone footpads had considerable inflammation by 48 hours after infection and treatment as seen in representative photomicrographs (Figure 5, C and D) and in quantitative scoring of cellular inflammation (Figure 5, I). Cellular inflammation 48 hours after treatment, as scored for $>20$ fields $(40 \times)$, was not significantly increased by the addition of trimannose-coated beads compared with $L$. major infection alone. As noted by the board-certified pathologist who scored these slides, footpads infected with L. major and treated with trimannose beads contained more polymorphonuclear cells, peaking at the 24hour time point (polymorphonuclear/mononuclear inflammatory cell ratio of 2.19:0.89), compared with the more characteristic mononuclear-predominant cell infiltrate seen with L. major infection alone.

Regarding the entry of CSCBs into phagocytic cells, trimannose-coated beads, observed as green spheroids (Figure 5, E-G), entered $\mathrm{CD} 11 \mathrm{~b}^{+}$cells rapidly within 2 hours of treatment (Figure 5J), whereas uncoated and lactose-coated beads more slowly entered $\mathrm{CD}_{11 \mathrm{c}^{+}}$cells (data not shown). Uncoated beads were not found to enter CD $11 b^{+}$cells to any significant amount compared with either sugar-coated bead (Figure 5J).

\section{Discussion}

\section{In Vivo Trimannose Beads Rapidly Enter Macrophages and Alter Cellular Inflammation in Response to L. major Infection}

Based on the present findings regarding alterations in cytokine production after treatment with trimannosecoated beads during L. major infection, we were interested in determining from these same animals which

\section{Immunomodulation by Pathogen Oligosaccharides}

Establishment of infection depends on the initial interaction between pathogen and host cell, interactions that are dictated by molecules and receptors expressed on the surface of each. Intracellular pathogens mediate their interactions through specific host cell receptors to facilitate host cell adherence and entry. ${ }^{31-33}$ Use of particular 
host-cell receptors and mode of entry are determinative in specifying the subsequent intracellular fate of the organism and in directing the innate immune response. ${ }^{31,36}$ The present novel pathogen-derived CSCBs ${ }^{19}$ allowed investigation of how sugars themselves alter in vitro and in vivo innate immune responses. ${ }^{37}$ We found that specific pathogen carbohydrate moieties could alter IL-12p40 production in vitro and in vivo (Figures $2 \mathrm{~B}$ and $4 \mathrm{~A}$ ), a cytokine that is critical for the induction of Th1 immune responses required to control Leishmania and Mycobacterium infection. We observed significant suppression of IL-12p40 production from macrophages when treated with trimannose-coated beads, a cap sugar found on ManLAM of M. tuberculosis (Figure 2A). We observed the opposite response, an increased number of DLN cells producing IL-12p40, after treatment with trimannose beads when used in vivo to alter the response of the murine innate immune response to cutaneous infection with $L$. major. We suggest that these differences between in vivo and in vitro results are likely to be due to different cell types induced to make IL12p40: macrophages in the present in vitro experiments versus whole DLN cell populations in vivo. Use of alternative lectin receptors as expressed on dendritic and $B$ cells versus macrophages may have led to these observed different cellular responses to trimannosecoated beads.

\section{Host Cell Lectin-Oligosaccharide Interactions}

We used blocking antibodies against the binding regions of TLR2 and MR to identify that each oligosaccharidecoated bead had different receptor interactions that lead to the production of IL-12p40 or, in the case of uncoated beads, noninteraction (Figures 3 and $4 \mathrm{~A}$ ). We propose that this lack of lectin-receptor interaction with uncoated latex beads leads to much slower uptake of uncoated beads into $\mathrm{CD} 11 \mathrm{~b}^{+}$or $\mathrm{CD}_{11 \mathrm{c}^{+}}$cells (Figure $5 \mathrm{~J}$; data not shown). This lack of receptor-mediated uptake would then hinder triggering of signaling cascades, which leads to proinflammatory cytokine production and influx of inflammatory cells, the overall effect of which dampened the immune response to $L$. major after treatment with uncoated latex beads (Figures 4 and 5). In contrast, signaling via TLR2 promoted proinflammatory cytokine production after trimannose-coated bead treatment (Figure 4B), which ultimately may have led to a more robust inflammatory response to $L$. major infection compared with the responses observed with $L$. major alone, control uncoated, or lactose-coated beads.

\section{TLR2 Signaling Based on Heterodimer Formation}

TLR2 can form heterodimers with TLR6 and TLR1. ${ }^{38-40}$ Herein, we demonstrated a dependence on TLR2 for alteration of proinflammatory cytokine production from in vitro macrophages and DLN cells of mice infected with $L$. major and treated with trimannose-coated beads (Figures
2 and 4). Based on previously published studies, we suggest that TLR2 may be forming a heterodimer with TLR1 versus TLR6 to promote the production of proinflammatory cytokines. ${ }^{41}$ This would be in contrast to the TLR2/6 heterodimer-dependent signaling demonstrated after cardiomyocyte infection with the kinetoplast family member to Leishmania, Trypanosoma cruzi. ${ }^{42}$ TLR2/1 heterodimer-based signaling triggered by trimannosecoated beads may have induced DLN proinflammatory cytokine production and led to overall changes in the innate inflammatory response to $L$. major infection and trimannose bead treatment.

\section{Pathogen Mechanisms for Deterrence of Oligosaccharide-Mediated Responses}

It has previously been shown that as expressed on metacyclic promastigote parasites, $L$. donovani LPG is likely to fold over, potentially "hiding" the cap sugars from immune surveillance. ${ }^{43,44}$ This pathogen adaptation may allow metacyclic parasites to be briefly present extracellularly after introduction into the host via the vector sand fly, protecting the parasite from complement lysis but also providing a reduced proinflammatory response than would otherwise occur without LPG glycocalyx folding. The trimannose cap on ManLAM has been shown to engage MR, prevent phagolysosomal maturation, ${ }^{45}$ and, thus, lead to similar reduced cytokine production. We demonstrated that trimannose-coated beads inhibited IL$12 p 40$ through an MR-mediated mechanism, which may help balance signaling mediated through other lectin receptors $^{36}$ and allow intracellular survival of $M$. tuberculosis in macrophages. ${ }^{46,47}$ In both instances, deception of the innate immune response, either by hiding inflammatory mediators as observed in Leishmania metacyclic promastigote LPG trimannose cap or preserving a better intracellular home through cap sugar-ligand-receptor binding as promoted by mycobacterial ManLAM trimannose caps, may allow pathogen persistence in an otherwise hostile immune environment.

Herein, we demonstrate, for the first time, the ability of pathogen-derived cap sugars alone to alter innate immune responses in vitro, after macrophage activation with strong stimulants (LPS and IFN- $\gamma$ ), and in vivo, after $L$. major infection. Trimannose cap sugar treatment with in vivo L. major infection promoted IL-12p40 and other Th1characterized cytokine production from mouse DLN cells known to improve the Th1 productive immune response to $L$. major infection. This cap sugar model system allowed comparison of four structurally different cap sugars derived from pathogens of variable virulence. A better understanding of how these carbohydrate moieties are recognized and how they alter the innate immune response reveals the importance of this complex group of abundantly expressed molecules found on almost all pathogens. Given the differences in these responses, further understanding of how simple pathogen-derived oligosaccharides alter macrophage function and presentation to $T$ cells is critical. The ability to use discreet oligosaccharides to alter the innate immune response 
will allow rational development of more effective vaccine adjuvants.

\section{Acknowledgments}

We thank Doug Jones and Katherine Gibson-Corley for helpful discussions.

\section{References}

1. Akira S: Pathogen recognition by innate immunity and its signaling Proc Jpn Acad Ser B Phys Biol Sci 2009, 85:143-156

2. Geijtenbeek TB, van Vliet SJ, Engering A, 't Hart BA, van Kooyk Y: Self- and nonself-recognition by C-type lectins on dendritic cells. Annu Rev Immunol 2004, 22:33-54

3. Janeway CA Jr, Medzhitov R: Innate immune recognition. Annu Rev Immunol 2002, 20:197-216

4. Geijtenbeek TB, Van Vliet SJ, Koppel EA, Sanchez-Hernandez M, Vandenbroucke-Grauls CM, Appelmelk B, Van Kooyk Y: Mycobacteria target DC-SIGN to suppress dendritic cell function. J Exp Med 2003, 197:7-17

5. Sacks D, Sher A: Evasion of innate immunity by parasitic protozoa Nat Immunol 2002, 3:1041-1047

6. Sacks DL, Modi G, Rowton E, Spath G, Epstein L, Turco SJ, Beverley SM: The role of phosphoglycans in Leishmania-sand fly interactions. Proc Natl Acad Sci U S A 2000, 97:406-411

7. Beverley SM, Turco SJ: Lipophosphoglycan (LPG) and the identification of virulence genes in the protozoan parasite Leishmania. Trends Microbiol 1998, 6:35-40

8. Dermine JF, Goyette G, Houde M, Turco SJ, Desjardins M: Leishmania donovani lipophosphoglycan disrupts phagosome microdomains in J774 macrophages. Cell Microbiol 2005, 7:1263-1270

9. Descoteaux A, Turco SJ: Glycoconjugates in Leishmania infectivity Biochim Biophys Acta 1999, 1455:341-352

10. Gaur U, Showalter M, Hickerson S, Dalvi R, Turco SJ, Wilson ME Beverley SM: Leishmania donovani lacking the Golgi GDP-Man transporter LPG2 exhibit attenuated virulence in mammalian hosts. Exp Parasitol 2009, 122:182-191

11. Thomas JR, McConville MJ, Thomas-Oates JE, Homans SW, Ferguson MA, Gorin PA, Greis KD, Turco SJ: Refined structure of the lipophosphoglycan of Leishmania donovani. J Biol Chem 1992, 267 6829-6833

12. Ilg T: Proteophosphoglycans of Leishmania. Parasitol Today 2000 16:489-497

13. Turco SJ, Spath GF, Beverley SM: Is lipophosphoglycan a virulence factor? a surprising diversity between Leishmania species. Trends Parasitol 2001, 17:223-226

14. Maeda N, Nigou J, Herrmann JL, Jackson M, Amara A, Lagrange PH Puzo G, Gicquel B, Neyrolles O: The cell surface receptor DC-SIGN discriminates between Mycobacterium species through selective recognition of the mannose caps on lipoarabinomannan. J Biol Chem 2003, 278:5513-5516

15. Naderer T, Vince JE, McConville MJ: Surface determinants of Leishmania parasites and their role in infectivity in the mammalian host Curr Mol Med 2004, 4:649-665

16. Guenin-Mace L, Simeone R, Demangel C: Lipids of pathogenic Mycobacteria: contributions to virulence and host immune suppression. Transbound Emerg Dis 2009, 56:255-268

17. Vercellone A, Nigou J, Puzo G: Relationships between the structure and the roles of lipoarabinomannans and related glycoconjugates in tuberculosis pathogenesis. Front Biosci 1998, 3:e149-e163

18. Mukbel R, Petersen CA, Jones DE: Soluble factors from Leishmania major-specific CD4(+)T cells and B cells limit L. amazonensis amastigote survival within infected macrophages. Microbes Infect 2006 8:2547-2555

19. Song EH, Osanya AO, Petersen CA, Pohl NL: Synthesis of multivalent tuberculosis and Leishmania-associated capping carbohydrates reveals structure-dependent responses allowing immune evasion. J Am Chem Soc 2010, 132:11428-11430
20. Kaiser E, Colescott RL, Bossinger CD, Cook PI: Color test for detection of free terminal amino groups in the solid-phase synthesis of peptides. Anal Biochem 1970, 34:595-598

21. Dubois M, Gilles KA, Hamilton JK, Rebers PA, Smith F: Colorimetric method for determination of sugars and related substances. Analytic Chem 1956, 28:350-356

22. Adams EW, Ratner DM, Seeberger PH, Hacohen N: Carbohydratemediated targeting of antigen to dendritic cells leads to enhanced presentation of antigen to T cells. Chembiochem 2008, 9:294-303

23. Nigou J, Zelle-Rieser C, Gilleron M, Thurnher M, Puzo G: Mannosylated lipoarabinomannans inhibit IL-12 production by human dendritic cells: evidence for a negative signal delivered through the mannose receptor. J Immunol 2001, 166:7477-7485

24. Heinzel FP, Schoenhaut DS, Rerko RM, Rosser LE, Gately MK: Recombinant interleukin 12 cures mice infected with Leishmania major J Exp Med 1993, 177:1505-1509

25. Reiner SL, Zheng S, Wang ZE, Stowring L, Locksley RM: Leishmania promastigotes evade interleukin 12 (IL-12) induction by macrophages and stimulate a broad range of cytokines from CD4+ T cells during initiation of infection. J Exp Med 1994, 179:447-456

26. Sacks D, Noben-Trauth N: The immunology of susceptibility and resistance to Leishmania major in mice. Nat Rev Immunol 2002 2:845-858

27. Zaph $\mathrm{C}$, Scott $\mathrm{P}$ : Interleukin-12 regulates chemokine gene expression during the early immune response to Leishmania major. Infect Immun 2003, 71:1587-1589

28. Trinchieri G: Interleukin-12 and the regulation of innate resistance and adaptive immunity. Nat Rev Immunol 2003, 3:133-146

29. Jiao X, Lo-Man R, Guermonprez P, Fiette L, Deriaud E, Burgaud S, Gicquel B, Winter N, Leclerc C: Dendritic cells are host cells for mycobacteria in vivo that trigger innate and acquired immunity. $\mathrm{J} \mathrm{Im}$ munol 2002, 168:1294-1301

30. Aguilar Torrentera F, Laman JD, Van Meurs M, Adorini L, Muraille E, Carlier Y: Endogenous interleukin-12 is critical for controlling the late but not the early stage of Leishmania mexicana infection in C57BL/6 mice. Infect Immun 2002, 70:5075-5080

31. Bhatt K, Salgame P: Host innate immune response to Mycobacterium tuberculosis. J Clin Immunol 2007, 27:347-362

32. Rhoades ER, Archambault AS, Greendyke R, Hsu FF, Streeter C, Byrd TF: Mycobacterium abscessus Glycopeptidolipids mask underlying cell wall phosphatidyl-myo-inositol mannosides blocking induction of human macrophage TNF- $\alpha$ by preventing interaction with TLR2. J Immunol 2009, 183:1997-2007

33. Takagi H, Furuya N, Kojima N: Preferential production of IL-12 by peritoneal macrophages activated by liposomes prepared from neoglycolipids containing oligomannose residues. Cytokine 2007, 40:241-250

34. Glatigny S, Blaton MA, Marin J, Mistou S, Briand JP, Guichard G Fournier C, Chiocchia G: Insights into spatial configuration of a galactosylated epitope required to trigger arthritogenic T-cell receptors specific for the sugar moiety. Arthritis Res Ther 2007, 9:R92

35. Singh SK, Stephani J, Schaefer M, Kalay H, Garcia-Vallejo JJ, den Haan J, Saeland E, Sparwasser T, van Kooyk Y: Targeting glycan modified OVA to murine DC-SIGN transgenic dendritic cells enhances MHC class I and II presentation. Mol Immunol 2009, 47:164-174

36. van Vliet SJ, den Dunnen J, Gringhuis SI, Geijtenbeek TB, van Kooyk $Y$ : Innate signaling and regulation of Dendritic cell immunity. Curr Opin Immunol 2007, 19:435-440

37. Song EH, Osanya AO, Petersen CA, Pohl NL: Synthesis of multivalent tuberculosis and Leishmania-associated capping carbohydrates reveals structure-dependent responses allowing immune evasion. J Am Chem Soc 2010, 132:11428-11430

38. Takeda K, Kaisho T, Akira S: Toll-like receptors. Annu Rev Immunol 2003, 9:9

39. Triantafilou M, Gamper FG, Haston RM, Mouratis MA, Morath S, Hartung T, Triantafilou K: Membrane sorting of toll-like receptor (TLR)2/6 and TLR2/1 heterodimers at the cell surface determines heterotypic associations with CD36 and intracellular targeting. J Biol Chem 2006, 281:31002-31011

40. Takeda K, Kaisho T, Akira S: Toll-like receptors. Annu Rev Immunol 2003, 21:335-376

41. Depaolo RW, Tang F, Kim I, Han M, Levin N, Ciletti N, Lin A, Anderson $D$, Schneewind O, Jabri B: Toll-like receptor 6 drives differentiation of 
tolerogenic dendritic cells and contributes to LcrV-mediated plague pathogenesis. Cell Host Microbe 2008, 4:350-361

42. Petersen CA, Krumholz KA, Burleigh BA: Toll-like receptor 2 regulates interleukin-1 $\beta$-dependent cardiomyocyte hypertrophy triggered by Trypanosoma cruzi. Infect Immun 2005, 73:6974-6980

43. Barron TL, Turco SJ: Quantitation of Leishmania lipophosphoglycan repeat units by capillary electrophoresis. Biochim Biophys Acta 2006 , 1760:710-714

44. Sacks DL, Pimenta PF, McConville MJ, Schneider P, Turco SJ: Stagespecific binding of Leishmania donovani to the sand fly vector midgut is regulated by conformational changes in the abundant surface lipophosphoglycan. J Exp Med 1995, 181:685-697

45. Kang PB, Azad AK, Torrelles JB, Kaufman TM, Beharka A, Tibesar E, DesJardin LE, Schlesinger LS: The human macrophage mannose receptor directs Mycobacterium tuberculosis lipoarabinomannanmediated phagosome biogenesis. J Exp Med 2005, 202:987-999

46. Cambi A, Figdor CG: Dual function of C-type lectin-like receptors in the immune system. Curr Opin Cell Biol 2003, 15:539-546

47. van Kooyk Y, Geijtenbeek TB: DC-SIGN: escape mechanism for pathogens. Nat Rev Immunol 2003, 3:697-709 